\title{
Heavy Rainfall and Risk of Infectious Intestinal Diseases in the Most Populous City in
}

\section{Vietnam}

Dung Phung ${ }^{1,3}$, Cordia Chu ${ }^{1}$, Shannon Rutherford ${ }^{1}$, Huong Lien Thi Nguyen ${ }^{2}$, Mai Anh Luong ${ }^{2}$, Cuong Manh Do ${ }^{2}$, Cunrui Huang ${ }^{3,1}$.

${ }^{1}$ Centre for Environment and Population Health, Griffith University, Australia

${ }^{2}$ Health Environment Management Agency, Vietnam Ministry of Health, Vietnam

${ }^{3}$ School of Public Health, Sun Yat-sen University, China

\section{Corresponding Author:}

Dung Phung

Phone: $\quad+61(07) 37355078$

Fax: $\quad+61(07) 37355318$

Email:_d.phung@griffith.edu.au

Address: Centre for Environment and Population Health Nathan campus, Griffith University

179 Kessels Road, Nathan

Brisbane, Queensland 4111, Australia

Cunrui Huang

Phone: $\underline{+86-20-87334211}$

Email: huangcr@mail.sysu.edu.cn

Address: School of Public Health, Sun Yat-sen University

No.74, Zhongshan Road \#2, Guangzhou 510080, CHINA 


\section{Abstract}

The association between heavy rainfall and infectious intestinal diseases (IID) has not been well described and little research has been conducted in developing countries. This study examines the association between heavy rainfall and hospital admissions for IID in Ho Chi Minh City, the most populous city in Vietnam.

An interrupted time-series method was used to examine the effect of each individual heavy rainfall event (HRE) on IID. The percentage changes in post-HRE level and trends of IID were estimated for 30 days following each HRE. Then a random-effect meta-analysis was used to quantify the pooled estimate of effect sizes of all HREs on IID. The pooled estimates were calculated over a 21 day lag period.

The effects of a HRE on IID varied across individual HREs. The pooled estimates indicate that the levels of IID following a HRE increased from $7.3 \%$ to $13.5 \%$ for lags from 0 to 21 days, however statistically significant increases were only observed for lags from 4 to 6 days (13.5\%,95\%Cl: 1.4-25.4; 13.3\%, 95\%Cl: 1.5-25.0; and 12.9\%, 95\%Cl: 1.6-24.1 respectively). An average decrease of $0.11 \%$ (95\%Cl:-0.55-0.33) per day was observed for the post-HRE trend.

This finding has important implications for the projected impacts on residents living in this city which is highly vulnerable to increased heavy rainfall associated with climate change. Adaptation and intervention programs should be developed to prevent this additional burden of disease and to protect residents from the adverse impacts of extreme weather events.

Keywords: heavy rainfall, intestinal infectious diseases, hospital admission, Vietnam 


\subsection{Introduction}

There is increasing evidence that on-going global climate change, which has been accelerated by anthropogenic greenhouse gas emissions over the last few decades, contributes to more extreme hydrologic events such as heavy rainfall, increased floods and droughts (IPCC 2013). Such extreme weather events are considered significant health hazards which cause increased morbidity and mortality (Ye et al. 2012;Basu and Ostro 2008). Heavy rainfall events have been found to be associated with an elevated risk of waterborne diseases in both developed (Curriero et al. 2001;Thomas et al. 2006;Nichols et al. 2009;Chou et al. 2010;Jagai et al. 2015;Wade et al. 2014) and developing countries (Adkins et al. 1987;Dewan et al. 2013;Seidu et al. 2013). However, the relationship between heavy rainfall events and hospital admissions for specific intestinal infectious diseases has not been well described in previous studies. Most studies have focussed on investigating the number of disease outbreaks following heavy rainfall events (Drayna et al. 2010) but have not examined the short-term changes of level and trend in actual number of individual illness cases following heavy rainfall events. Moreover, the link between heavy rainfall and infectious intestinal diseases (IID) such as cholera, typhoid, salmonella and other bacteria has rarely been analysed in developing settings which have a high population density and fewer resources to cope with the potentially growing impacts of climate change related extreme weather events.

Ho Chi Minh City (HCMC) is the most populous city in Vietnam where urban floods have emerged as a serious concern due to the city's rapid growth. Despite the construction and continued expansion of the urban drainage system to address the demands of increased population and infrastructure, this system is often overloaded as water levels in the river, rainfall and the runoff ratio have increased over the past decades (Phi 2008). Rapid population growth, shortage of investment and aging water and sewage infrastructure have resulted in alarmingly polluted waterways and exposed the inhabitants of HCMC to considerable risk (Ha et al. 2008; Vo 2007; Wust et al. 2002). The water of the Saigon and Dong Nai rivers is used as the source of drinking water for residents of HCMC, which has limited purification and control over industrial and domestic waste discharge (Hong et al. 2000; Vo 2007). Vo (2007) estimated that only $40 \%$ of waste water from industrial and hospital sources discharged into the Saigon River is treated. Exacerbating this problem is HCMC's highly vulnerability to climate change (Yusuf and Francisco 2009). Extreme weather 
events such as heavy rainfall and associated floods have become more frequent in HCMC recently, leading to substantial economic and social losses. For example, research has indicated a linear increasing trend of yearly max $180 \mathrm{~mm}$ rainfall events with an overall increasing trend in rainfall of about $0.8 \mathrm{~mm}$ per year over the past 50 years (Phi 2008). The phenomena of heavy rainfall and related inundation may cause negative effects on residents' health, especially intestinal infectious illness due to the possible distribution of infectious agents following heavy rainfall and related floods. However, investigation of the relationship between heavy rainfall and adverse health effects in the context of climate change has seldom been conducted, resulting in a lack of understanding of the potential future impacts associated with increasing extreme rainfall events and hence a lack of evidence for developing appropriate strategies to reduce health risks in this the largest city in Vietnam.

This study examines the association between heavy rainfall events and hospital admissions for intestinal infectious diseases in HCMC, Vietnam.

\subsection{Methods}

The study design incorporates interrupted time-series and meta-analysis methods using daily rainfall and hospital admission data.

\subsection{Study area}

The study was conducted in Ho Chi Minh City (HCMC). HCMC is located in southern Vietnam, about $1730 \mathrm{~km}$ from the capital city, Hanoi. HCMC has a total area of 2,095.5 km², including 19 urban and 5 suburban districts and a total population of $8,224,4008.4 \%$ of the population of Vietnam) and a population density of 3,900 persons $/ \mathrm{km}^{2}$ (HCMC SO 2016). $\mathrm{HCMC}$ has a tropical climate with an average annual rainfall of 1,800 $\mathrm{mm}$ (about 150 rany days per year). It has two distinct seasons: the rainy season (May-October) and the dry season (December - October). The average temperature is $28^{\circ} \mathrm{C}$, and the average humidity is 78-82\% (ADB 2014). Supplement 1 shows a photo of flooding in HCMC after a heavy rainfall event. In the South-East region, where HCMC is located, infectious and parasitic diseases are leading causes of morbidity with $10.5 \%$ of hospital admissions, and of mortality with 13.1\% of admissions (HSY 2013). Of these diseases, Norovirus (NoV) is a leading cause of acute infectious intestinal disease among children less than 5 years old. It has been estimated that $20.6 \%$ of children hospitalized with acute diarrhoea in Ho Chi Minh City 
tested positive for NoV (Phan et al. 2013). Recent studies have shown that diarrhoeal diseases remain a considerable public health problem in $\mathrm{HCMC}$ and these diseases are associated with seasonality and climatic conditions such as flooding, air temperature, and humidity (Thompson et al. 2015a;Thompson et al. 2015b).

HCMC has a total of 72 hospitals that are classified by level (national, city, and district) and speciality (general or multi-faculty, and specialized hospitals e.g. paediatric hospital).

\subsection{Data collection}

Data on hospital admissions for intestinal diseases (ICD-10 codes: A00-A09) were extracted from hospital admission records from February 2004 to December 2013 in the two largest hospitals in HCMC, Gia Dinh People's Hospital and 115 People's Hospital. These hospitals, which have 1200 and 1600 patient beds respectively, were selected from four big multi-faculty hospitals in HCMC. These hospitals are at the highest level of public multifaculty hospitals administered by the HCMC Health Department and serve patients from all districts across HCMC with 1000-2000 inpatients daily. These hospitals also serve people outside of the city in case of emergency or for self-funded services.

Data extracted from the hospital admissions were categorized by primary and discharge diagnoses, date of admission, date of discharge, age, sex, and address of individual patients (street and district only). The authors selected A00-A09 codes rather than the symptoms' or non-specific codes for two reasons. First, symptoms are not specific to intestinal diseases only. Second, patients sometime tend to self-treat and don't go to hospitals if they just have symptoms, since in Vietnam people can buy medicine in pharmacies without a doctor's prescription. Thus, we would miss all of these cases if we considered symptoms as the outcome of the study. In this study, the authors included only residents of $\mathrm{HCMC}$ and excluded all cases who were not $\mathrm{HCMC}$ residents or who were transferred from hospitals located in other cities/provinces. The address described in the hospital records was used for this purpose.

Daily meteorological data were collected from the Southern Regional HydroMeteorological Centre (SRHMC) for the February 2004 to December 2013 period. The data were recorded from one hydro-meteorological station located in the central district of HCMC (longitude, $106^{\circ} 39^{\prime} 59.75$ East: latitude, 1047'47.48 North), the Tan Son Hoa Meteorological station, which monitors air temperature, surface temperature, humidity, precipitation, sunlight hours, wind direction and speed, cloud and other climatic conditions. 
The data are managed by SRHMC as the lead agency for hydro-meteorological information in the South of Vietnam. The data collected for this study, comprised: daily minimum, maximum, and average temperatures $\left({ }^{\circ} \mathrm{C}\right)$ and minimum, maximum and average relative humidity (\%), and daily cumulative rainfall $(\mathrm{mm})$.

\subsection{Data analysis}

Two phases were involved in the data analysis. First, we examined the influence of each heavy rainfall event (HRE) on hospital admissions for intestinal infectious diseases. Secondly, we used a random-effect meta-analysis to estimate the pooled effect size of all heavy rainfall events on intestinal infectious disease admissions (IID).

An interrupted time-series method using a segmented regression analysis (Poisson family) was used to examine the HRE-individual effect on IID. Consistent with a previous study by Dratyna et al (2010), a heavy rainfall event was defined as daily cumulative rainfall $>=95^{\text {th }}$ percentile for $>=2$ consecutive days. The percentage change in level ( $y$-intercept) and trend (the slope) of IID was estimated for 30 days after each heavy rainfall event, which was coded as 1 (HRE) or 0 (no HRE) in the regression model. Due to the variety in intensity and length of each HRE, and potential for adaptation of residents to HRE events such as household and community actions to cope with extreme rainfall events (e.g. moving furniture up to high places, preparation of foods and drugs, installation of backwater valves to proactively reduce inundated flood, community implementation plans, preparedness of health centres to reduce outbreak of diseases following HE events, etc.)(Guilbault 2015), at Stage 1 of the data analysis we distinguished the effect of every individual HRE to avoid the diluted effects of exposures (HRE events) and proposed to examine heterogeneity in effects of these HRE events. Moreover, interrupted time-series analysis requires excluding the effect of the previous event while computing the effect of the current event. For instance, the HRE event 1 (code as 1 ) was excluded from the variable of HRE while calculating the effect of HRE event 2. In order to model the effects of multi-HRE and to avoid incorrect specification of HRE effects, we excluded from the analysis daily IID that occurred during the lag or 'during the event' period. Thus, the IID for day 0 and day 1 were excluded when estimating the impact of HRE on day 3. The possible delayed effects of HRE on IID were examined for lags of up to 21 days. The climate factors (temperature and humidity), which are potential confounding factors and have been reported as having a non-linear effect on 
IA (Phung et al. 2015a; Phung et al. 2015b), were controlled using a natural cubic spline function with 3 degrees of freedom. The day-of-week effect was also controlled using dummy variables for each day of the week. Equation 1 identifies the model fitted.

$$
\begin{aligned}
& Y_{t} \sim \operatorname{Poisson}\left(\mu_{t}\right)
\end{aligned}
$$

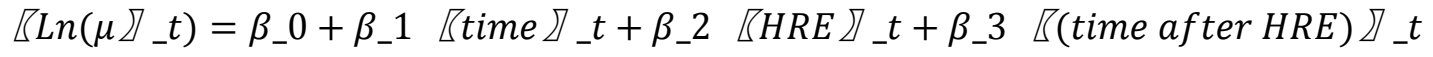

$$
\begin{aligned}
& +s(\text { Temperature, } 3 d f)+s(\text { Humidity, } 3 d f)+\gamma D O W
\end{aligned}
$$

where, $Y_{t}$ is the mean of daily counts of IID; time $_{t}$ is a continuous variable indicating time in days at time $t$ from the start of the observation period; $H R E_{t}$ is an indicator for time $t$ occurring before ( $\mathrm{HRE}=0)$ or after $(\mathrm{HRE}=1)$ the $\mathrm{HRE}$; time after $H R E$ is a continuous variable counting the number of days (up to 30 ) after the HRE at time $t ; s$ is the natural cubic spline functions of daily temperature and humidity with 3 degrees of freedom; and $D O W$ is day of the week. In this model, $\beta_{1}$ is the estimated change in the daily IID that occurs with each day before the HRE (i.e. baseline trend); $\beta_{2}$ is the estimated level change in the mean of daily IID immediately after $t$ days following the HRE date; and $\beta_{3}$ is the estimated change in the trend in the mean of daily IID after the HRE compared with the daily trend before the HRE.

The meta-analysis technique is used to calculate a pooled estimate of effect size of the results obtained from multiple studies but it can also be used to compute a pooled estimate of effect size of the results from multiple events, monitoring or interventions within one study. For example, the study by Milojevic et al (2012) used meta-analysis to evaluate the health effects of multiple flooding events in rural Bangladesh. Phung et al (2015) used metaanalysis to compute the pooled estimate of health effects of high temperature from multiple provinces in the whole Mekong Delta Region. In this study, we used meta-analysis to quantify the impact of HRE on IID by computing the pooled estimate of effect sizes from all individual HREs. Because the impact of the HRE might vary with events due to the variability of $\mathrm{HRE}$ intensity and duration, resulting in considerable heterogeneity of findings, a random-effect meta-analysis was applied to calculate within-HRE and between-HRE variation and generate pooled estimates of changes in level and trend (\% change) of IID. Then the result can generalize the effect of a HRE event. The pooled estimates were calculated for each day after the HRE over 21 days (lag 21). Heterogeneity between HREs was quantified by the coefficient of inconsistency (I-Squared) which describes the 
percentage of total variation across provinces that is due to heterogeneity (Higgins et al. 2003).

Data management, transformation, and analyses were conducted using Stata 11.0 (Stata Corporation, College Station, TX, USA).

\subsection{Results}

\subsection{Descriptive statistics}

Descriptive statistics of IID and climate factors are shown in Figure 1. During the study period, there were a total of 54,077 hospital admissions for intestinal infectious diseases with a mean of 15 admissions per day (SE, 0.1). The total number of IID almost doubled from $2004(3,767)$ to $2012(6,746)$ (t-test for trend, $p$-value<0.01). The total number of rainy days was 1,570 days with an average of 160 days per year. The $95^{\text {th }}$ percentile of daily cumulative rainfall was $30.8 \mathrm{~mm}$ for the whole year and $37.9 \mathrm{~mm}$ for the wet season only (MayNovember). The number of heavy rainfall events (HRE), defined as $>=95^{\text {th }}$ for two or more consecutive days, was 12 for the entire study period. Figure 1 shows that the pattern of daily IID corresponds visually to the variation of cumulative rainfall during the study period. The average daily mean temperature in $\mathrm{HCMC}$ was $28.2^{\circ} \mathrm{C}(\mathrm{SE}, 0.02)$, with a minimum of 23 ${ }^{\circ} \mathrm{C}$ and maximum of $32^{\circ} \mathrm{C}$. The average relative humidity recorded was $74.4 \%(\mathrm{SE}, 0.1)$ with a daily minimum of $51 \%$ and daily maximum of $97 \%$.

\subsection{Association between heavy rainfall and admissions for infectious intestinal diseases}

Supplement 2 presents details of the changes in level and trends of each individual HRE at different lags, and Figure 2 shows an example of these changes in one HRE event. The post-HRE changes in level and trend of IID varied with HREs and lag days (Supplement 2). For the same day as HRE occurrence (lag 0), the baseline trend of IID before HREs was subtly but non-significantly changed with a range of $-0.015 \%-0.03 \%$ per day. In contrast, positive increases in level of IID following a HRE were observed in 8 out of the 12 HREs, of which significant increases in IID were found following 5 HREs, with a range of 19\% - 44\% (25 Sept. 2004, 28\%; 02 Sept. 2007, 31\%; 22 July 2008, 23\%; 04 Oct. 2010, 44\%; and 18 Oct. 2010, 19\%). The 30 -day post-HRE trend of IID ranged from $-1.7 \%$ to $1 \%$ per day across HREs, of which decreased trends in IID were observed in 5 out of 12 HREs. Figure 2 shows an example of the changes in admissions due to IID following a HRE (2-3 Sept. 2007). The effects of HRE on IID for other lags from 1 to 21 days are fully reported in Supplement 2. 
Across HREs, significant increases in post-HRE levels of IID were more frequent for lags of 46 days across all HREs in comparison with other lags.

The pooled estimate of baseline trend indicates that IID increased negligibly at a rate of $0.017 \%$ (95\% Cl: $0.012-0.022)$ per day prior to HREs. On average a heavy rainfall event was associated with anincrease from $7.3 \%$ to $13.5 \%$ for lags $0-21$ days, however statistically significant increases were only observed for lags 4-6 days (Day 4, 13.5\%,95\%Cl: 1.4-25.4; Day 5, 13.3\%, 95\%Cl: 1.5-25.0; Day 6, 12.9\%, 95\%Cl: 1.6-24.1; and Day 10, 12.5, 95\%Cl: 0.624.5). Figure 3 shows the pooled estimates of levels of IID, which slowly decreased from lag 7 to lag 21 days. The pattern of changes in baseline, post-HRE level and trend was modelled using the pooled estimates of lag 4 , as this was the most statistically significant lag. Figure 4 shows this modelling pattern with the rates of changes in baseline trend $(0.02 \%, 95 \% \mathrm{Cl}$ : 0.01-0.02), post-HRE level (13.4\%, 95\% Cl: $1.4-25.3)$ and post-HRE trend $(-0.11 \%, 95 \% \mathrm{Cl}:-$ 0.55-0.33). The pooled estimates of change in level of IIDs calculated for each lag from 0 to 21 are shown in Supplements 3 and 4 . The percentages of total variation across HRE events caused by heterogeneity were high for most of the lag-day analysis $\left(1^{2}, 78 \%-84 \%\right)$. The heterogeneity indicates that the effects observed are highly variable and inconsistent across HRE events.

\subsection{Discussion}

This is the first study to comprehensively examine the short-term pattern of change in hospitalization due to intestinal infectious diseases in relation to heavy rainfall events (HREs) in Vietnam. The univariate analysis indicated that the overall trend of IID has increased from 2004 to 2012. Although no studies have investigated reasons for this increasing trend of infectious intestinal diseases from 2004 to 2012, some potential reasons include increased population and population density, rapid urbanization, better health surveillance systems to identify cases and to link database and report systems from hospitals and preventive medicine centres or deterioration in water quality. At this study design meants that we examined the change of IID risk over a short period of time (3 weeks) prior and post HRE, the increase observed in admissions over the 8-10 year study priod are not likely bias the results. The study used interrupted time-series and meta-analysis to examine the effects of multiple HREs on IID and modelled the pattern for HRE-IID relationship. During the study period 12 HREs were identified. The pooled estimates indicate 
that on average, a heavy rainfall event was significantly associated with increases in hospital admissions for intestinal infectious diseases, although the magnitude of HRE-IID association varied for individual HREs. The modelled pattern of the overall HRE-IID relationship reveals an average increase of $13.2 \%$ in IID observed $4-6$ days after a HRE.

The positive association between heavy rainfall and intestinal infectious disease admissions found in this study is consistent with the evidence reported from previous studies. A recent systematic review by Levy et al (2016) found that $71 \%$ of articles reported a significant association between heavy rainfall and diarrhoea, and this positive association was observed in both developing (Dewan et al. 2013;Seidu et al. 2013;Adkins et al. 1987; Hashizume et al. 2007) and developed countries (Curriero et al. 2001; Thomas et al. 2006; Chou et al. 2010;Nichols et al. 2009; Rose et al. 2000). A study in the USA (Drayna et al. 2010) also found that any rainfall four days prior was significantly associated with an $11 \%$ increase in acute hospital visits due to infectious intestinal diseases. Several studies found that heavy rainfall after a dry period was associated with increased diarrhoea (Carlton et al. 2014;Adkins et al. 1987; Nichols et al. 2009). However, this modified effect was not observed in our study, since almost all the heavy rainfall events during the study period occurred in the middle of wet seasons during the study period. A few studies focusing on the relationship between climate factors (including rainfall) and diarrhoea have been conducted in southern Vietnam (Few et al. 2013; Kien et al. 2010;Kelly-Hope et al. 2008;Phung et al. 2015a;Phung et al. 2015b). These studies suggest that diarrhoea is associated with disruption to normal water/sanitation services and hygiene behaviour, and with the spreading of contamination by flood events after heavy rainfall, which can contaminate drinking water sources and inundate latrines and reduce access to safe water sources and affect hygienic toilet practices. However, none of these studies have as comprehensively described the short-term pattern of intestinal risk associated with heavy rainfall events as this study. The high heterogeneity of the effects across HRE events observed in this study may be due to variation in HRE characteristics (intensity, length, spatial distribution of HRE impacts), or to socio-economic conditions and demographic factors related to health adaptive capacity, such as preparedness of preventive medicine and healthcare system and individual household coping strategies, to such heavy rainfall events in specific communities. Further studies that consider these factors should be conducted to better identify the cause of variability in the effects of HREs. 
We propose several possible pathways for infection from HREs. First, HREs can mobilize pathogens from human/animal excreta present in soils and on environmental surfaces or sub-surfaces and transport them into surface water exposing inhabitants to infectious agents (Levy et al. 2016). Further treatment of water sources during flood events in HCMC can be compromised. Second, some studies have also indicated that HREs can also lead to resuspension of pathogens in sediment or in soils (Jofre et al. 2010;Jean et al. 2006), and cause contamination of groundwater (Gelting et al. 2005), leading to saturation of the subsurface, which facilitates water transport of pathogens into surface or ground water (Fong et al. 2007). Third, HRE can promote manure-borne oocyst transport of protozoan pathogens in agricultural settings (Sterk et al. 2013) and may result in splashing of manureamended soils onto fresh produce (Liu et al. 2013) hence increasing health risks through food consumption. Exposure to zoonotic pathogens can also generate illness through recreational contact such as swimming (Ihekweazu et al. 2006). In this study, the disruption of water/sanitation is likely more than zoonotic transmission, since in the urban areas of HCMC high population density and overload of water systems may result in the disruption of continuity of water supply and sanitation facilities. Meanwhile, the transmission of zoonotic pathogens is likely more common in rural areas with more agricultural activities (e.g. animal stocks) and natural recreational contact (e.g. swimming in natural ponds, rivers). A more comprehensive overview of potential mechanisms for the patterns of the effects of HRE and flooding on diarrhoeal diseases is described in the review by Levy et al (2016).

Our study has some limitations. First, the data on hospital admissions for intestinal infectious diseases were collected from only two hospitals in the HCMC and represent the most serious cases that were required to be hospitalized. Minor cases are not likely to be admitted to hospitals, hence reducing the number of admissions making data analyses less sensitive in detecting the relationship between HRE and IID. However, the two hospitals involved in this study are the biggest multi-faculty hospitals in HCMC and patients were admitted from all around HCMC, so the pattern of IID in these two hospitals would be representative for IID in HCMC. Second, the socio-economic and behavioural factors of individual cases, which might cause variability in HRE-IID relationship across geographical areas and communities, were not considered in this study. However the study examined the short-term relationship between HRE and IID such that the socio-economic and behavioural factors are not expected to change in such a short period of time. Unfortunately, we did not 
have data of disease outbreaks, so future studies should consider this as one factor to control. Third, this study could not examine the relationship between HRE and individual cause-specific IID due to the very small number of admissions for each disease from 2 hospitals of a single city. A previous study has indicated that the effects of climate factors may be different for specific types of diseases (Phung et al. 2016). Thus, future studies on a larger scale with multiple cities should be conducted to increase the number of cases for cause-specific IID. Finally, this study was unable to collect data on the spatial impact of HRE events which may lead to misclassification of spatial distribution of exposures. For example, for a HR event might have greater impacts in some districts might be heavily due to more exposed to rainfall. Thus the study assumed that all HRE events had the same impact on the entire city. Future study should consider adjusting the data on spatial coverages or intensity of rainfall events. In order to better develop appropriate adaption strategies, further study should be conducted to better understand the contamination pathways of the pathogens causing IID among residents.

\subsection{Conclusion}

The results of this study indicate that heavy rainfall events significantly increase the risk of hospitalization for intestinal infectious diseases in Ho Chi Minh City. As HCMC is highly vulnerable to climate change and extreme rainfall events are projected to increase in this city these findings suggested that there is likely to be an increased hospital and community burden from IID in the future. Thus our findings help us better understand the projected impacts on residents in this most populous city of Vietnam. Public health adaptation and intervention programs (e.g. improving the capacity of the health sector in the field of climate change adaptation, educating community members in minimizing the effects of extreme weather events, developing HRE-health risk early warning systems, etc., improving treatment systems continuously and in anticipating of HRE, consuming alternative water sources during HREs) should be developed to prevent this additional burden of disease and to protect residents from changing climate conditions. In addition, the evidence from this study may also imply that similar effects will be observed in other cities which have conditions similar to HCMC. Future studies may need to be conducted to understand the HRE-IID relationship on a larger scale across Vietnam. 


\section{Acknowledgement}

DP was supported by a Griffith University Post-doctoral Fellowship 2015-2016. DP and CH were supported by the APN is Asia-Pacific Network for Global Change Research (CRRP201610MY-Huang).

Competing of Interest: No 


\section{References}

Adb 2014. Vietnam: Ha Noi and Ho Chi Minh City Power Grid Development Sector Project.

Adkins H.J., Escamilla J., Santiago L.T., Ranoa C., Echeverria P. \& Cross J.H. 1987. Two-year survey of etiologic agents of diarrhoeal disease at San Lazaro, Manila, Republic of the Philippines. J. Clin. Microbiol, 25, 1143-1147.

Basu R. \& Ostro B.D. 2008. A multicountry analysis identifying the populations vulnerable to mortality associated with high ambient temperature in California. Am J Epidemiol, 168, 632637.

Chou C., Wu J., Wang Y., Huang H., Sung F. \& Chuang C. 2010. Modelling the impact of climate availability on diarrhea-associated diseases in Taiwan (1996-2007). Science of the Total Environment, 409, 43-51.

Curriero F.C., Patz J., Rose J. \& Lele S. 2001. The association between extreme precipitation and waterborne disease outbreaks in the United States, 1948-1994. Am J Public Health, 91, 11941199.

Dewan A.M., Corner R., Hashizume M. \& Ongee E.T. 2013. Typhoid Fever and Its Association with Environmental Factors in Dhaka Metropolitant Area of Bangladesh: A Spatial and Time-Series Approach. Plos Neglected Tropical Diseases, 7, e1998.

Drayna P., Mclellan S.L., Simpson P., Li S. \& Gorelick M.H. 2010. Association between rainfall and pediatric emergency department visits for acute gastrointestinal illness. Environmental Health Perspective, 118, 1439-1443.

Few R., Lake I., Hunter P.R. \& Tran P.G. 2013. Seasonality, disease and behaviour: Using multiple methods to explore socio-environmental health risks in the Mekong Delta. Social Science \& Medicine, 80, 1-9.

Fong T.T., Mansfiled L.S., Wilson D.L., Schwab D.J., Molloy S.L. \& Rose J.B. 2007. Massive microbiological groundwater contamination associated with a waterborne outbreak in Lake Erie, South Bass Island, Ohio. Environmental Health Perspective, 115, 865-864.

Gelting R., Sarisky J., Selman C., Otto C., Higgins C., Bohan P.O., et al. 2005. Use of a systems-based approach to an environmental health assessment for a waterborne disease outbreak investigation at a snowmobile lodge in Wyoming. Int. J. Hyg. Environ. Health, 208, 67-73.

Guilbault S. 2015. Adapting to Extreme Rainfall: The Institute for Catastrophic Loss Reduction celebrates leadership amongst Canadian cities in its latest publication. IN: www.blouindunn.com. Acessed Date: November 2016.

Ha N.T.V., Kitajima M., Hoang N.V.M., Matsubara K., Takizawa S., Katayama H., et al. 2008. Baterial contamination of raw vegetables, vegetable-related water and river water in Ho Chi Minh City, Vietnam. Water Sci. Technol., 58, 2403-2411.

Hashizume M., Armstrong B., Wagatsuma Y., Faruque A.S.G., Hayashi T. \& Sack D.A. 2007. Rotavirus infections and climate variability in Dhaka, Bangladesh: a time-series analysis. Epidemiol. Infect., 136, 1281-1289.

Hcmc So 2016. Statistical Office in Ho Chi Minh City. IN: www.pso.hochiminhcity.gov.vn.

Higgins J.P.T., Thompson S.G., Deeks J.J. \& Altman D.G. 2003. Measuring inconsistency in metaanalyses. BMJ, 327, 557-560.

Hong L., Slooten K.B., Sauvain J., Minh T.L. \& Tarradellas J. 2000. Toxicity of Sediments from the Ho Chi Minh City Canals and Saigin River, Viet Nam. Environ. Toxicol., 15, 469-475.

Hsy 2013. Viet Nam Health Statistic Yearbook.

Ihekweazu C., Barlow M., Roberts S., Christensen H., Guttridge B., Lewis D., et al. 2006. Outbreak of E.coli $\mathrm{O} 157$ infection in the south west of the UK: risks from streams crossing seaside beaches. Euro Surveill, 11, 128-130.

Ipcc 2013. Intergovernmental Panel on Climate Change (IPCC, 2013) Climate Change 2013: The physical science basis. www.ipcc.ch. Accessed 16/06/2015.

Jagai J.S., Li Q., Wang S., Messier K.P., Wade T.J. \& Hilborn E.D. 2015. Extreme Precipitation and Emergency Room Visits for Gastrointestinal Illnes in Areas with and without Combined 
Sewer Systems: An Analysis of Massachusets Data, 2003-2007. Envi Health Persp, 123, 873878.

Jean J.S., Guo H.R., Chen S.H., Liu C.C., Chang W.T., Yang Y.J., et al. 2006. The association between rainfall rate and occurrence of an enterovirus epidemic due to a contaminated well. J. Appl. Microbiol., 101, 1224-1231.

Jofre J., Blanch A.R. \& Lucena F. 2010. Water-borne infectious disease outbreak associated with water scarcity and rainfall events. In Water Scarcity in the Mediterranean: Perspectives Under Global Change, 8 ed.; Sabater, S.; Barcelo, D., Eds; Springer-Verlag: Berlin 2010; pp 147-159.

Kelly-Hope L.A., Alonso W.J., Vu T.D., Do G.C., Dang A.D., Lee H., et al. 2008. Temporal Trends and Climatic Factors Associated with Bacterial Enteric Diseases in Vietnam, 1991-2001. Envi Health Persp, 116, 7-12.

Kien T.M., Tran T.T.H., Cuong H.D. \& Shaw R. 2010. IDENTIFYING LINKAGES BETWEEN RATES AND DISTRIBUTIONS OF MALARIA, WATER-BORN DISEASES AND INFLUENZA WITH CLIMATE VARIABILITY AND CLIMATE CHANGE IN VIETNAM. In: SHAW, R., PULHIN, J. M. \& PEREIRA, J. J. (eds.) Climate Change Adaptation and Disaster Risk Reduction: An Asian Perspective.

Levy K., Woster A.P., Goldstein R.S. \& Carlton E.J. 2016. Untangling the impacts of climate change on waterborne diseases: A systematic review of relationship between diarrhoeal diseases and temperature, rainfall, flooding, and drought. Environmental Science \& Technology.

Liu C., Hofstra N. \& Franz E. 2013. Impacts of climate change on the microbial safety of pre-harvest leafy green vegetables as indicated by Escheriachia coli 0157 and Samonella spp. Int. J. Food Microbiol., 163, 119-128.

Nichols G., Lane C., Asgari N., Verlander N.Q. \& Charlett A. 2009. Rainfall and outbreaks of drinking water related disease and in England and Wales. J Water Health, 7, 1-8.

Phan V.T.M., Thompson C., Hoang P.L., Pham T.N.T., Ha V., Nguyen H.M.V., et al. 2013. Endemic Norovirus Infectious in Children. Ho Chi Minh City, Vietnam, 2009-2010. Emerging Infectious Diseases, 19, 977-980.

Phi H.L. Impacts of Climate Changes and URbanization on Urban Inundation in Ho Chi Minh City. 11th International Conference on Urban Drainage, Edinburgh, Scotland, UK, 2008.

Phung D., Guo Y., Nguyen H.T.L., Rutherford S., Baum S. \& Chu C. 2016. High temperature and risk of hospitalizations, and effect modifying potential of socio-economic conditions: A multiprovince study in the tropical Mekong Delta Region. Environment International, 92-93, 7786.

Phung D., Huang C., Rutherford S., Chu C., Wang X., Nguyen M., et al. 2015a. Association between climate factors and diarrhoea in a Mekong Delta area. Int J Biometeorol, 2014.

Phung D., Huang C., Rutherford S., Chu C., Wang X., Nguyen M., et al. 2015b. Temporal and spatial patterns of diarrhoea in the Mekong Delta area, Vietnam. Epidemiol. Infect., 2015, 1-10.

Rose J.B., Daeschner S., Easterling D.R., Curriero F.C., Lele S. \& Patz J.A. 2000. Climate and waterborne disease outbreaks. J Am Water Works Assoc, 92, 77-87.

Seidu R., Stenstrom T.A. \& Lofman O. 2013. A comparative cohort study of the effect of rainfall and temperature on diarrhoeal disease in faecal sludge and non-faecal sludge applying communities. J. Water Clim. Change, 4, 90-102.

Sterk A., Schijven J., Nijs T.D. \& Husman A.M.D. 2013. Direct and Indirect Effects of Climate Change on the Risk of Infection by Water-Transmitted Pathogens. Environ. Sci. Technol., 47, 1264812660.

Thomas M.K., Charron D.F., Waltner-Toews D., Schuster C., Maarouf A.R. \& Holt J.D. 2006. A role of high impact weather events in waterborne disease outbreaks in Canada, 1975-2001. International Journal of ENvironmental Health Research, 16, 167-180.

Thompson C.N., Phan V.T.M., Nguyen M.V., Pham M.V., Nguyen V.T., Cao T.T., et al. 2015a. A prospective multi-center observational study of children hospitalized with diarrhoea in Ho Chi Minh City Vietnam. Am. J. Trop. Med. Hyg., 95, 1045-1052. 
Thompson C.N., Zelner J.L., Tran N.H.D., Phan M.V.T., Hoang P.L., Nguyen H.T., et al. 2015b. The impact of environmental and climatic variation on the spatiotemporal trends of hospitalized pediatric diarrhoea in Ho Chi Minh City, Vietnam. Health \& Place, 35, 147-154.

Vo P.L. 2007. Urbanization and water management in Ho Chi Minh City, Vietnam - issues, challenges and perspective. GeoJournal, 70, 75-89.

Wade T.J., Lin C.J., Jagai J.S. \& Hilborn E.D. 2014. Flooding and emergency room visits for gastrointestinal illness in Massachusets: a case-crossover study. PloS One, 9, e110474.

Wust S., Bolay J. \& Du T.T.N. 2002. Metropolization and the Ecological Crisis: Precarious Settlements in Ho Chi Minh City, Vietnam. Environ. Urban., 14, 211-224.

Ye X., Wolff R., Yu W., Vaneckova P., Pan X. \& Tong S. 2012. Ambient temperature and morbidity: a review of epidemiological evidence. Envi Health Persp, 120, 19-27.

Yusuf A.A. \& Francisco H. 2009. Climate Change Vulnerability Mapping for Southeast Asia. Economy and Environment Program for Southeast Asia (EEPSEA). In: http://www.eepsea.org. 
Figure1. Daily admissions due to infectious intestinal diseases, and rainfalls, temperature, humidity Figure2. Changes in admissions due to infectious intestinal diseases following a heavy rainfall event Figure3. Pooled estimates of $\%$ change in admissions due to infectious intestinal diseases by lag days Figure4. Change in baseline, level, and trend of admissions due to infectious intestinal diseases in 4days following a heavy rainfall event

\section{Supplement}

1. Flooding following a HRE in HCMC

2. Effects of individual HRE

3. Forest plots of pooled estimates from lag 1-12

4. Forest plots of pooled estimates from lag 13-21 